\title{
1 Sexual dimorphism in the Drosophila metabolome increases throughout
}

2 development

3

$4 \quad$ FC Ingleby* and EH Morrow

5

6 Evolution, Behaviour and Environment Group, School of Life Sciences, University

7 of Sussex, John Maynard Smith Building, Falmer, Brighton, UK

8

$9 \quad *$ Corresponding author:

10 Email: f.ingleby@sussex.ac.uk

11 Telephone: +44 (0) 1273678559

12

13 Keywords: Drosophila melanogaster; metabolome; ontogeny; sexual dimorphism;

14 transcriptome

15 


\section{ABSTRACT}

18 The expression of sexually dimorphic phenotypes from a shared genome

19 between males and females is a longstanding puzzle in evolutionary biology.

20 Increasingly, research has made use of transcriptomic technology to examine the

21 molecular basis of sexual dimorphism through gene expression studies, but even

22 this level of detail misses the metabolic processes that ultimately link gene

23 expression with the whole organism phenotype. We use metabolic profiling in

24 Drosophila melanogaster to complete this missing step, with a view to examining

25 variation in male and female metabolic profiles, or metabolomes, throughout

26 development. We show that the metabolome varies considerably throughout

27 larval, pupal and adult stages. We also find significant sexual dimorphism in the

28 metabolome, although only in pupae and adults, and the extent of dimorphism

29 tends to increase throughout development. We compare this to transcriptomic

30 data from the same population and find that the general pattern of increasing sex

31 differences throughout development is mirrored in RNA expression. We discuss

32 our results in terms of the usefulness of metabolic profiling in linking genotype

33 and phenotype to more fully understand the basis of sexually dimorphic

34 phenotypes. 


\section{Introduction}

Sexual dimorphism is common across a wide range of plant and animal species, and there is a longstanding field of research examining how the sexes can differ so markedly when they share the majority of their genes (Darwin 1871; Lande 1980). Most recently, research has built on the premise that for sexually dimorphic phenotypes to develop from the same genes, there is likely to be sex differences at the molecular level (Ellegren and Parsch 2007). As such, a proliferation of data on sex-biased gene expression has shed some light on the molecular basis for sex differences. Sex-biased gene expression has been found in a diverse range of species - but especially in model insect species where highthroughput -omic technologies are increasingly cheap and available - and the extent of sexual dimorphism at the level of the transcriptome can be large (reviewed by Ingleby et al. 2015).

Some research has also examined how sex differences in gene expression progress throughout development. At a phenotypic level, it is generally the case that sexual dimorphism increases throughout development, ultimately resulting in highly dimorphic adult phenotypes that are adapted to sex-specific reproductive roles. Research has shown that this progression through development is often mirrored by transcriptomic sex differences. In Drosophila melanogaster, for instance, sex-biased gene expression is more prevalent in adults than in pre-adult stages, as demonstrated by Ingleby et al. (2016) and is also clear from comparisons of pre-adult data (e.g. Perry et al. 2014) with adults (e.g. Innocenti and Morrow 2010). Similarly, the extent of sex-biased gene expression has been shown to increase throughout development in the mosquito, Anopheles gambiae (Magnusson et al. 2011), and the silk moth, Bombyx mori (Zhao et al. 2011). However, there is a considerable gap between gene expression and the wholeorganism phenotype, with a series of cellular and metabolic processes linking gene to phenotype. This may be particularly relevant in a developmental context, where the expression of a gene at a particular stage could act to trigger a 
69 pathway where the phenotypic effect might only be measurable at a later stage.

70 This highlights the potential significance of the processes that link the genotype

71 and phenotype. Here, we examine this gap by quantifying the metabolic profile,

72 or metabolome, of male and female D. melanogaster at three stages throughout

73 development (i.e. larvae, pupae and adults).

74

The usefulness of this approach is highlighted by research that illustrates the sizeable gap between genotype and phenotype - generally only a small fraction of phenotypic variation is thought to be explained by genetic variation, whereas over $50 \%$ of metabolic variation can be explained by genetic variation (Suhre et al. 2011). Drosophila melanogaster has been cited as a particularly useful insect model for metabolomic studies (e.g. Chintapalli et al. 2013), and recent studies have identified many interesting patterns of metabolome variation in this species: for example, variation across the sexes (Hoffmann et al. 2014) and with age (Sarup et al. 2012, Hoffmann et al. 2014), as well as metabolic plasticity to various environmental factors as adults (Overgaard et al 2007, Colinet et al. 2012, Laye et al. 2015, Williams et al. 2015) and as larvae (Kostal et al. 2011).

We used GCMS analysis to identify and quantify compounds in the $D$. melanogaster metabolic profile from male and female samples of larvae, pupae and adults. Our results show that there is significant metabolome variation both throughout development and across sexes. In addition, we find that the extent of sexual dimorphism in the overall metabolic profile increases throughout development, and that this pattern is broadly mirrored in transcriptomic data from previous research. We discuss these results in terms of how metabolic profiling could be a useful tool for further research linking genotype to phenotype. 


\section{Materials and methods}

98

99

100

101

102

103

104

105

106

107

108

109

110

111

112

113

114

115

116

117

118

119

120

121

122

123

124

125

126

127 All samples were immediately flash frozen in liquid nitrogen, then processed

128 following Hoffmann et al. (2014) by thoroughly homogenising the sample with a

129

\subsection{Fly stocks}

Drosophila melanogaster samples for metabolic profiling were sampled from the established ' $\mathrm{LH}_{\mathrm{M}}$ ' population that has been reared in consistent laboratory conditions for more than 500 generations. This population has been maintained as a large outbred population with overlapping generations, using a standard molasses diet at $25^{\circ} \mathrm{C}, 65 \%$ relative humidity, and a $12: 12 \mathrm{~h}$ light:dark incubator light cycle.

\subsection{Sample collection and processing}

Male and female flies from the stock population were given $48 \mathrm{~h}$ to interact and mate, before males were removed, and females transferred to fresh vials of lightly yeasted food to lay eggs. Females laid eggs in these vials for $2 \mathrm{~h}$ before being transferred to fresh vials, and then there were two subsequent $2 \mathrm{~h}$ laying periods in fresh vials at 4 and 7 days later. This process created 3 sets of staged vials with developing offspring. For each set of vials, larvae were sexed at 4 days after laying by visual inspection under a dissecting microscope. At this point, developing testes can be clearly seen through the larval body wall of males, allowing male and female offspring to be separated into sex-specific vials to continue development. Ten larvae were counted per vial to standardise the rearing environment. Samples were collected 11 days after the initial laying vials were set up. At this point, third instar larvae, pupae and 1-day old virgin adults (unable to mate as they eclosed in sex-specific vials) were collected from each vial. Three individuals were pooled into an eppendorf vial from each of 4 replicate vials per developmental stage and sex $(\mathrm{N}=24$ independent replicates split equally across 2 sexes x 3 developmental stages). pestle motor in $150 \mu \mathrm{l}$ acetonitrile in water $(2: 1 \mathrm{v} / \mathrm{v})$. Samples were then 
130 centrifuged at $12,000 \mathrm{rpm}$ for 20 minutes, and the $100 \mu \mathrm{l}$ of supernatant pipetted

131 into a fresh eppendorf. Samples were stored at $-80^{\circ} \mathrm{C}$ before being analysed

132 approximately one week later. This involved loading the samples randomly into

133 a chilled autosampler, and injecting $20 \mu \mathrm{l}$ of sample into a GCMS (Agilent

$1346890 / 5973$ ) fitted with a DB-5MSUI column of $30 \mathrm{~m} \times 0.25$ internal diameter $\mathrm{x}$

$1350.25 \mu \mathrm{m}$ film thickness. Hydrogen was used as a carrier gas. The inlet was set at

$136280^{\circ} \mathrm{C}$ and the injection was in split mode. Separation of the extract was

137 optimised with a temperature cycle that held at $50^{\circ} \mathrm{C}$ for $1 \mathrm{~min}$, then increased at

$13810^{\circ} \mathrm{C} \mathrm{min}^{-1}$ to $320^{\circ} \mathrm{C}$. Integration of metabolite peaks was carried out using GC

139 ChemStation software (Agilent version B.04.02.SP1), but a clear signal could not

140 be detected for one male adult replicate, so the full analysis comprises $\mathrm{N}=23$

141 samples in total. Across all samples, 25 peaks were quantified and identified

142 using mass spectroscopy data in the AMDIS software v.2.71 (Table 1). Of these

143 compounds, 14 were present in both sexes and all stages. This indicates a

144 considerable degree of qualitative variation in metabolome throughout

145 development, but as our analyses focus on quantitative variation, the analysis

146 uses only the 14 common peaks as identified in Table 1.

\subsection{Data handling and analysis}

150 All analyses were carried out in R v.3.2.1. Data from the integrated peaks of all 25

151 compounds listed in Table 1 were used to calculate standardised peak areas

152 using a centred log ratio transformation on proportional peak areas

153 (Pawlowsky-Glahn and Buccianti 2011), as follows:

$$
\operatorname{standardised}_{n}=\ln \frac{\operatorname{prop}\left(\text { trait }_{n}\right)}{\left(\prod_{n=1}^{k} \operatorname{prop}\left(\text { trait }_{n}\right)\right)^{1 / 2}}
$$

157 where the divisor is the geometric mean of the proportional area of all $k$ traits

158 and the numerator is the proportional area of the $n^{\text {th }}$ trait. By using all peaks for 159 the standardisation calculation, and then filtering data afterwards to leave only 160 the 14 compounds expressed in both sexes and all stages, this avoids the 
Table 1. Full list of compounds identified via GCMS. The last three columns indicate whether the compound was present in each developmental stage, where ' $m$ ' indicates presence in male samples, ' $\mathrm{f}$ ' indicates presence in female samples, and ' $\mathrm{fm}$ ' indicates presence in both sexes. There is clear qualitative variation in metabolic profile throughout development. The analyses here aimed to examine quantitative variation only, and therefore used data only from compounds found in both sexes in all three developmental stages (shaded).

\begin{tabular}{|l|l|l|l|l|}
\hline \multirow{2}{*}{ Compound } & \multicolumn{4}{|l}{ Stage } \\
\cline { 3 - 5 } & Group & Larvae & Pupae & Adults \\
\hline Lactic acid & intermediate & $\mathrm{m}$ & $\mathrm{fm}$ & $\mathrm{fm}$ \\
\hline Alanine & amino acid & $\mathrm{fm}$ & $\mathrm{fm}$ & $\mathrm{fm}$ \\
\hline Valine & amino acid & $\mathrm{fm}$ & $\mathrm{fm}$ & $\mathrm{fm}$ \\
\hline Glycerine & intermediate & $\mathrm{fm}$ & $\mathrm{fm}$ & $\mathrm{fm}$ \\
\hline Leucine & amino acid & - & $\mathrm{fm}$ & $\mathrm{fm}$ \\
\hline Glycine & amino acid & - & $\mathrm{fm}$ & $\mathrm{fm}$ \\
\hline beta-Alanine & amino acid & - & - & $\mathrm{fm}$ \\
\hline Pyroglutamic acid & intermediate & - & $\mathrm{fm}$ & $\mathrm{fm}$ \\
\hline Glutamic acid & amino acid & - & $\mathrm{fm}$ & - \\
\hline Citric acid & intermediate & $\mathrm{fm}$ & $\mathrm{fm}$ & $\mathrm{fm}$ \\
\hline Inositol & polyol & $\mathrm{fm}$ & $\mathrm{fm}$ & $\mathrm{fm}$ \\
\hline Fructose & sugar & - & $\mathrm{fm}$ & - \\
\hline Methyl-malonic acid & intermediate & - & $\mathrm{fm}$ & - \\
\hline Glucose & sugar & $\mathrm{fm}$ & $\mathrm{fm}$ & $\mathrm{fm}$ \\
\hline Lysine & amino acid & - & - & $\mathrm{fm}$ \\
\hline Ribonic acid & intermediate & - & $\mathrm{fm}$ & - \\
\hline Palmitic acid & fatty acid & $\mathrm{fm}$ & $\mathrm{fm}$ & $\mathrm{fm}$ \\
\hline Uric acid & intermediate & - & $\mathrm{fm}$ & - \\
\hline Butyl palmitate & fatty acid & $\mathrm{fm}$ & $\mathrm{fm}$ & $\mathrm{fm}$ \\
\hline Linoleic acid & fatty acid & $\mathrm{fm}$ & $\mathrm{fm}$ & $\mathrm{fm}$ \\
\hline Oleic acid & fatty acid & $\mathrm{fm}$ & $\mathrm{fm}$ & $\mathrm{fm}$ \\
\hline Stearic acid & facid & $\mathrm{fm}$ & $\mathrm{fm}$ & $\mathrm{fm}$ \\
\hline Butyl stearate & $\mathrm{fm}$ & $\mathrm{fm}$ \\
\hline
\end{tabular}


bioRxiv preprint doi: https://doi.org/10.1101/073148; this version posted September 2, 2016. The copyright holder for this preprint (which was not certified by peer review) is the author/funder. All rights reserved. No reuse allowed without permission.

\begin{tabular}{|l|l|l|l|l|}
\hline Undecane & hydrocarbon & $\mathrm{fm}$ & $\mathrm{fm}$ & $\mathrm{fm}$ \\
\hline Cholesterol & intermediate & $\mathrm{fm}$ & $\mathrm{fm}$ & $\mathrm{fm}$ \\
\hline
\end{tabular}


161 problem of the zero-sum constraint of full rank data using this transformation. In

162 total, data on expression of 14 compounds was used in further analysis in order

163 to examine quantitative variation in metabolic profile across sexes and

164 development.

166 The analyses employ a combination of univariate and multivariate approaches in

167 order to examine variation in metabolism both as an overall metabolic profile as

168 well as for individual compounds. Exploratory initial analyses involved

169 hierarchical clustering of the samples based on a distance matrix using 'hclust'

170 and 'dist' functions in the R package 'stats'. The same clustering methods were

171 also carried out for the 14 metabolite compounds.

173 Next, univariate linear models were used to directly test for sex and stage

174 variation in expression of each of the 14 compounds individually. These linear

175 models used Bayesian inference within the 'MCMCglmm' package v2.22.1

176 (Hadfield 2010) and each took the basic structure:

$$
Y \sim S * D+\varepsilon
$$

where the response variable, $Y$, represents the standardised peak area for a given compound, $S$ is a fixed 2-level factor defining sex, $D$ is a fixed 3-level factor representing developmental stage, and $\varepsilon$ accounts for residual error variation. All models assumed a normal distribution and this assumption was checked for all compounds, as were model checks for Markov chain mixing and autocorrelation.

185 Models used a flat prior distribution and were ran for 100,000 iterations, with a 10,000 burn-in and a thinning interval of 25 . Significant differences in compound expression were inferred where $95 \%$ credible interval estimates from the posterior distribution for each sex and stage combination were non-overlapping.

189 Note that although the main results shown are based on these Bayesian analyses, 190 the equivalent frequentist linear models produce qualitatively identical results 191 (Table S1). 
Table S1. Results of non-Bayesian univariate linear models testing for differences in metabolite expression between developmental stages, sexes, and the interaction. For each effect, the F statistic and associated P value are shown. P values are corrected for FDR $<0.05$ and significant results are highlighted in bold. Models (described in the text) were equivalent to the Bayesian univariate linear models, and all results are qualitatively the same through Bayesian inference (shown in main text). Note that the interaction effect was significant for alanine and citric acid prior to FDR correction.

\begin{tabular}{|c|c|c|c|c|c|c|}
\hline \multirow[b]{2}{*}{ Compound } & \multicolumn{2}{|c|}{ Stage $x$ sex } & \multicolumn{2}{|l|}{ Stage } & \multicolumn{2}{|l|}{ Sex } \\
\hline & $\mathbf{F}$ & $\mathbf{P}$ & $\mathbf{F}$ & $\mathbf{P}$ & $\mathbf{F}$ & $\mathbf{P}$ \\
\hline Alanine & 3.45 & 0.25 & 3.60 & 0.13 & 5.81 & 0.18 \\
\hline Valine & 1.04 & 0.87 & 0.74 & 0.96 & 0.57 & 0.83 \\
\hline Glycerine & 0.43 & 0.87 & 7.07 & 0.02 & 1.90 & 0.60 \\
\hline Citric acid & 5.09 & 0.13 & 236.90 & $<0.001$ & 3.03 & 0.46 \\
\hline Inositol & 0.22 & 0.87 & 0.36 & 0.96 & 0.53 & 0.83 \\
\hline Glucose & 0.25 & 0.87 & 105.58 & $<0.001$ & 1.66 & 0.60 \\
\hline Palmitic acid & 0.27 & 0.87 & 0.12 & 0.96 & 0.05 & 0.99 \\
\hline Butyl palmitate & 0.42 & 0.87 & 0.12 & 0.96 & 0.57 & 0.83 \\
\hline Linoleic acid & 0.22 & 0.87 & 1.03 & 0.88 & 0.01 & 0.99 \\
\hline Oleic acid & 0.23 & 0.87 & 0.12 & 0.96 & 0.03 & 0.99 \\
\hline Stearic acid & 0.26 & 0.87 & 0.28 & 0.96 & 0.01 & 0.99 \\
\hline Butyl stearate & 0.44 & 0.87 & 0.04 & 0.96 & 0.37 & 0.85 \\
\hline Undecane & 15.06 & 0.002 & 19.35 & $<0.001$ & 7.43 & 0.18 \\
\hline Cholesterol & 0.07 & 0.93 & 0.07 & 0.96 & 0.01 & 0.99 \\
\hline
\end{tabular}


193 Sexual dimorphism in the overall metabolic profile was examined via linear

194 discriminant analysis, where differentiation between male and female samples

195 was modelled as a function of all 14 compounds. From the results of this model,

196 each sample was given a score along the discriminant function vector defining

197 maleness/femaleness, and these scores were modelled using the approach

198 described for equation [2], where $Y$ in this instance is the discriminant function

199 score for each sample.

200

201 Finally, variation in metabolic profile across sexes and development was

202 compared to variation in gene expression found across the same sample types,

203 derived from the same D. melanogaster population, in terms of gene expression.

204 This data is taken from a previous study of the population, where RNA-

205 sequencing was carried out on male and female samples of larvae, pupae and

206 adults (Ingleby et al. 2016). Here, we filtered the transcriptome to focus on a

207 subset of 26 genes that were identified as involved with the tricarboxylic acid

208 (TCA) cycle, using the database of D. melanogaster genes in the R package

209 'biomaRt' (filtered using 'grep' for the term 'TCA' in the gene description field).

210 This filter was applied based on the results of the metabolite analysis, which

211 showed interesting patterns of expression for compounds involved in the

212 tricarboxylic acid cycle. After filtering the RNA expression data to only include

213 these genes, a linear discriminant analysis was carried out exactly as described

214 for the metabolite data, and the resulting scores were modelled as before. 


\section{3. Results}

217

218 Initial hierarchical clustering of samples indicated strong differentiation

219 between developmental stages, with all samples grouped by stage (Figure 1).

220 Larval and pupal branches were more closely linked, with the adult branch as an

221 out-group. Within each stage, male and female samples did not cluster

222 separately, although arguably there was more evidence of sex differentiation in

223 adults than in the earlier developmental stages, since the adult male samples

224 clustered together (Figure 1).

226 Cluster analysis of the 14 metabolites that were found in all sample types (as

227 described in Table 1) showed a tendency for fatty acids and fatty acid derivatives

228 to group together (Figure 2), suggesting that these compounds were expressed

229 more similarly to each other than the other compounds analysed (predominantly

230 sugars and amino acids).

232 Significant differences in the expression of individual compounds across sexes

233 and developmental stages were tested directly via linear model analysis. Highly

234 significant differentiation across stages was found for glycerine, citric acid,

235 glucose and undecane (Figure 3), with further evidence of a significant sex $\mathrm{x}$

236 stage interaction in the expression of undecane. Visual inspection of the

237 posterior distribution estimates in Figure 3 also suggests a possible sex x stage

238 interaction for alanine and citric acid expression, but overlap between the 95\%

239 credible intervals for the different sample types shows that these interactions

240 are non-significant. Note that it is likely that relatively small sample sizes have

241 contributed to wide credible intervals (indicating wide variance around the

242 posterior estimates).

244 Despite non-significant sex differences in the univariate analyses, multivariate

245 analyses provide convincing evidence for sex differentiation between males and

246 females of the metabolic profile overall. A linear discriminant analysis clearly

247 differentiated between the sexes. This model was used to project samples onto

248 the linear discriminant vector LD1, giving each sample a score along an axis 


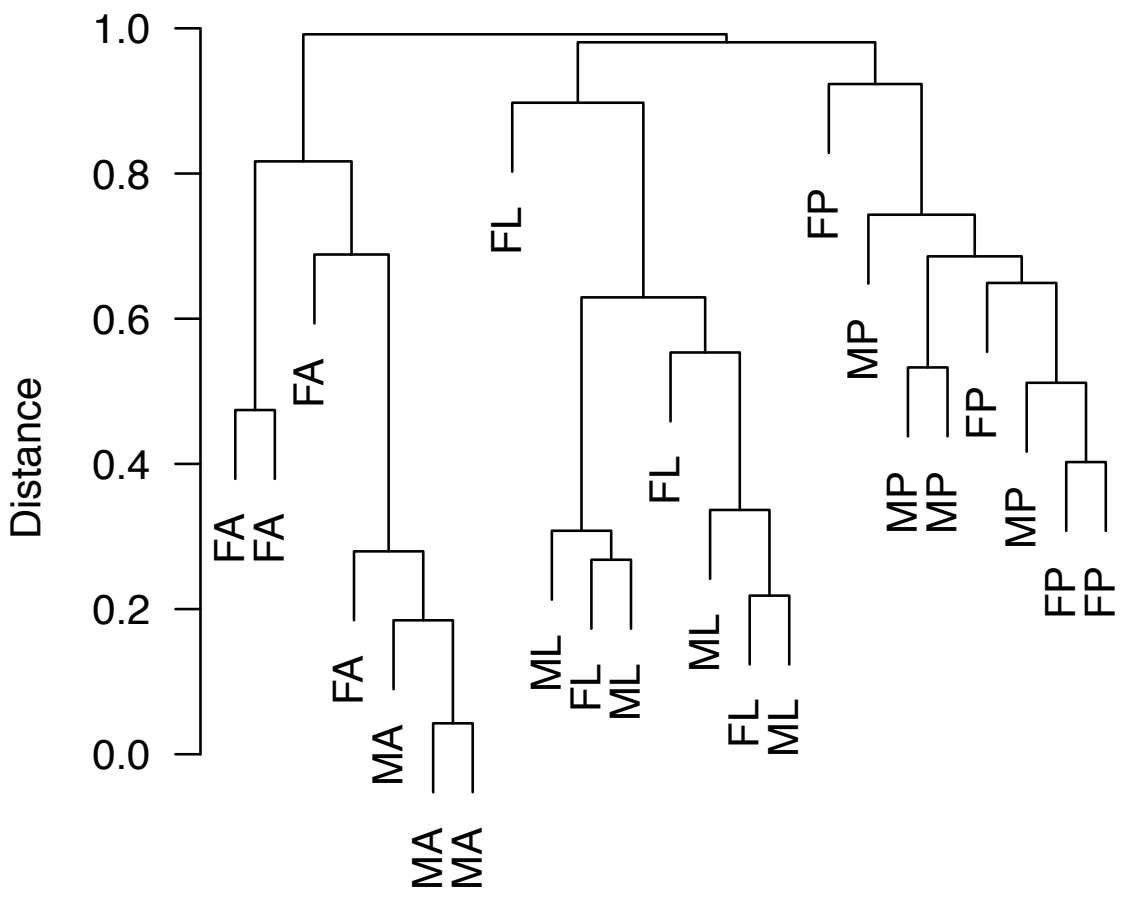


Figure 1. Result of hierarchical distance clustering to differentiate between samples. The distance is shown on the y-axis scale, and length of branches corresponds to the distance. The tree is based on expression of 14 compounds that were present at some level in all samples. 


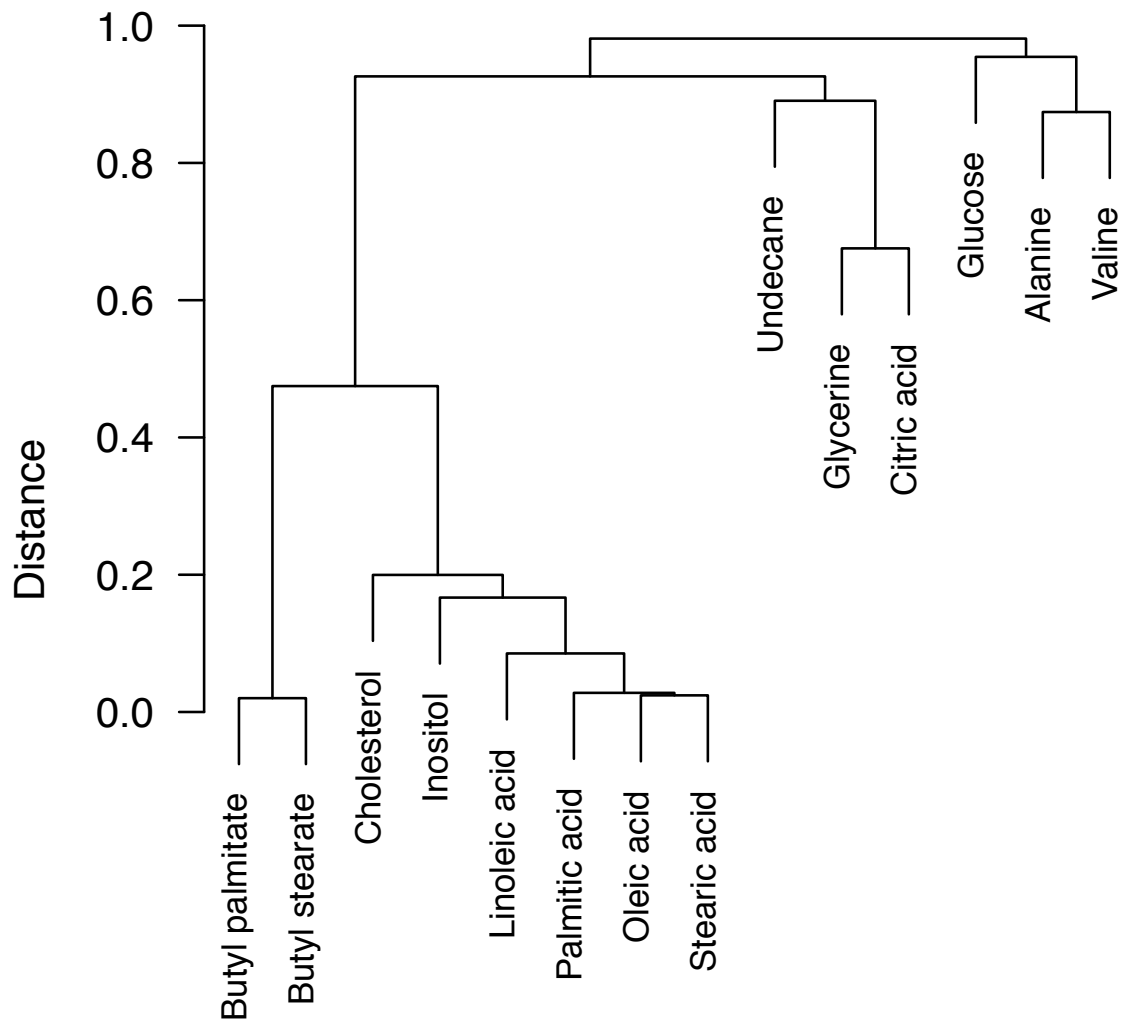


Figure 2. Result of hierarchical distance clustering to differentiate between metabolite compounds. The distance is shown on the $y$-axis scale, and length of branches corresponds to the distance value. All compounds were present at some level in all samples included in this analysis. 


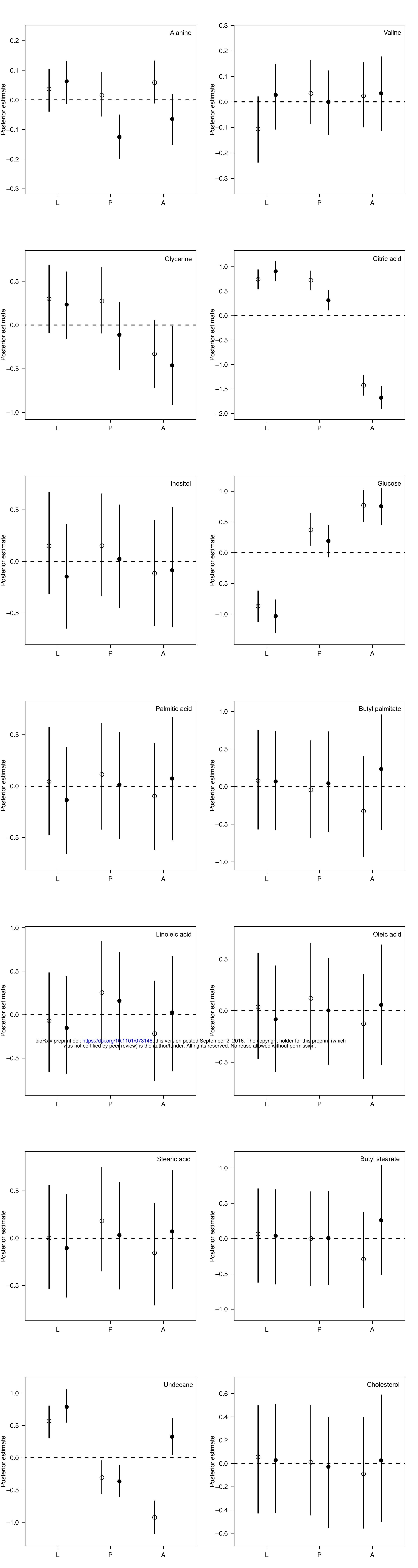


Figure 3. Posterior estimates (mean with $95 \%$ credible intervals) for each sex and stage combination taken from univariate MCMCglmm linear models for each of the 14 compounds (named in the top right of each plot). Female estimates are shown with open points, male estimates with filled points. $\mathrm{L}=$ larvae; $\mathrm{P}=$ pupae; and $\mathrm{A}=$ adults. 
249 describing metabolomic sex differentiation. These scores were significantly

250 different between male and female pupae and adults, although not between male

251 and female larvae (Figure 4). In addition, the difference between male and

252 female posterior means tends to increase throughout development (absolute

253 difference in posterior mean male and female scores in larvae $=1.34$; pupae $=$

2543.01 ; and adults $=3.85$ ), suggesting an increase in the extent of metabolome

255 sexual dimorphism from larvae through to adults.

256

257 The equivalent multivariate analysis based on gene expression data of 26 genes

258 associated with the tricarboxylic acid cycle (see Methods) revealed a very similar

259 pattern in sexual dimorphism of the transcriptome throughout development.

260 Scores for LD1 in this case were significantly different between males and

261 females at all three developmental stages (Figure 5), and the difference between

262 male and female posterior mean estimates tends to increase throughout

263 development (absolute difference in posterior mean male and female scores in

264 larvae $=4.73 ;$ pupae $=5.43$; and adults $=6.41$ ). 


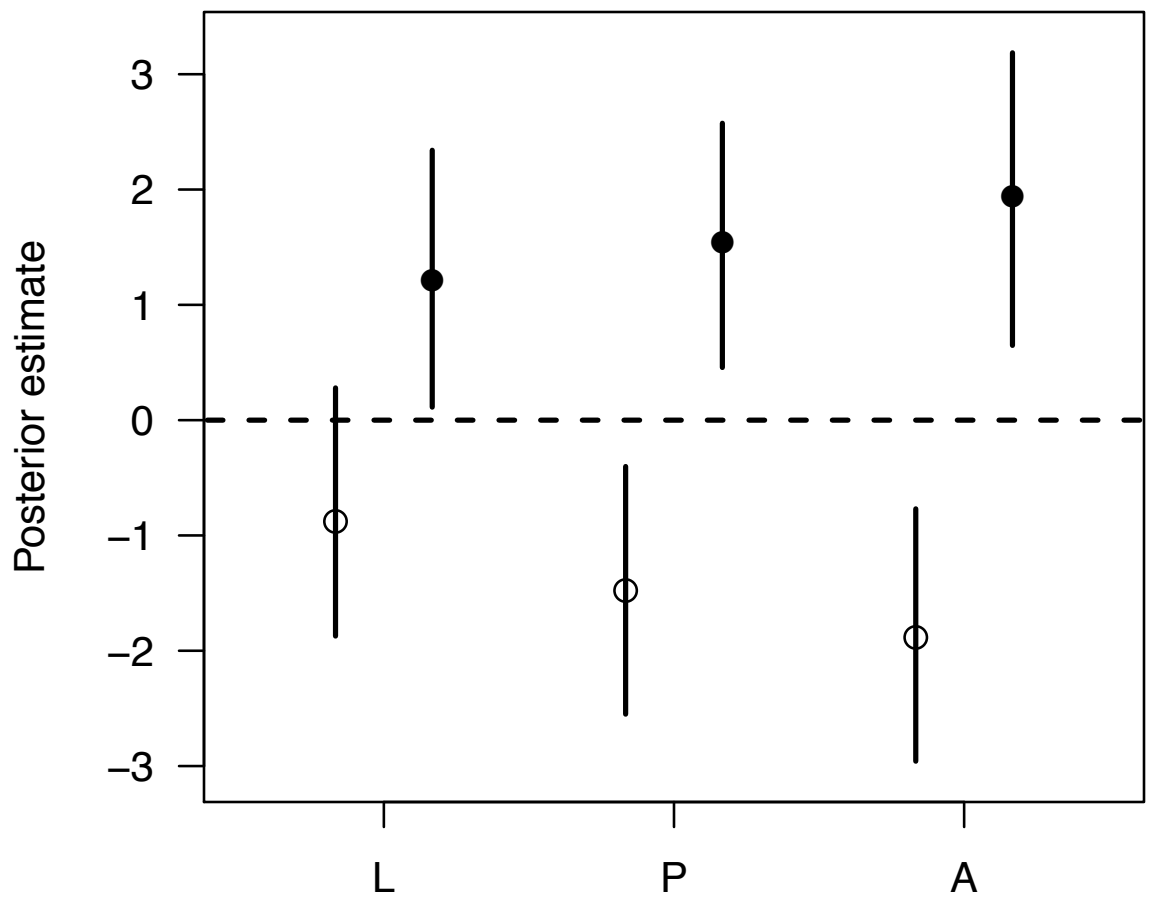


Figure 4. Posterior estimates (mean with 95\% credible intervals) for females (open points) and males (filled points) from each developmental stage for the first linear discriminant function (LD1). These values represent scores along a discriminant vector that differentiates between the sexes. 
Figure 5. Posterior estimates (mean with 95\% credible intervals) for females (open points) and males (filled points) from each developmental stage for the first linear discriminant function (LD1) based on RNA data from 26 genes identified as associated with the tricarboxylic acid cycle. These values represent scores along a discriminant vector that differentiates between the sexes. 


\section{Discussion}

268 Recent research has found variation in the D. melanogaster metabolome across 269 different environments, ages, and sexes (e.g. Colinet et al. 2012, Hoffmann et al. 270 2014, Laye et al. 2015, Williams et al. 2015). In this study, we found clear

271 variation in metabolic profile across larval, pupal and adult developmental 272 stages, as well as sex differences in the overall profile that appear to increase 273 throughout development. Additionally, we present some evidence that suggests 274 sex differences in the metabolome throughout development are mirrored by 275 ontogenetic patterns in the sex-biased expression of related genes. Our analyses 276 focussed on the evidence for quantitative variation in metabolites, but we also 277 found considerable qualitative variation throughout development, since almost 278 half of the individual metabolites identified in our samples were stage- or sex279 specific. This qualitative variation is not examined beyond identification here, 280 but may be of interest for future research.

Evidence for quantitative metabolome variation across development was very clear both from the multivariate analysis of the overall metabolic profile, as well as from univariate analysis of individual metabolites. Three of the metabolites that varied significantly between developmental stages - glucose, citric acid and glycerine - are key components in the tricarboxylic acid cycle, or Krebs cycle, that is largely responsible for providing cells with energy (Baldwin and Krebs 1981).

288 Generally, our data shows an increase in glucose throughout development, 289 combined with a decrease in citric acid and glycerine. While these patterns are interesting, it is difficult to disentangle any functional significance without a more detailed dataset, so here we simply note that the significant variation in these chemical components indicates that the dynamics of this cycle may vary throughout development. More generally, developmental variation in

294 metabolism has been found previously related to diapause in various insect species (Hahn and Denlinger 2007, Michaud and Denlinger 2007, Li et al. 2015, Dean et al. 2016), and Callier et al. (2015) found that metabolic responses to anoxia differed between larvae and adults in D. melanogaster. 
299 The strong differences between stages of these specific metabolites directed our

300 analysis of the transcriptome data, which focussed on a subset of 26 genes that

301 were associated with the cell tricarboxylic acid cycle. In fact, from the gene

302 descriptions in the 'biomaRt' database, many of these genes code for

303 dehydrogenase enzymes directly involved with different steps of the cycle. This

304 analysis showed an increasing extent of sexual dimorphism in gene expression

305 through development, and this supports the idea that these metabolites and the

306 associated genes could be interesting candidates for further research into sex

307 and stage differences in the metabolome.

309 The patterns of sex dimorphism in the RNA data mirrored those that we found

310 from the multivariate analysis of the overall metabolomic profile, although none

311 of the individual metabolites tested significantly for sex dimorphism in the

312 univariate analyses. In part, these non-significant results could be due to a

313 relatively small sample size, which would mean that power to detect differences

314 would be low, and this is supported by the wide intervals on the posterior

315 estimates. Indeed, sex differences in various aspects of metabolism have been

316 identified in previous studies of animals as diverse as D. melanogaster

317 (Hoffmann et al. 2014) and humans (Kochhar et al. 2006), and so the lack of

318 significant sex dimorphism for individual metabolites here is unexpected.

319 However, the multivariate profiling approach revealed sexual dimorphism in the

320 overall metabolome in the later stages of development (pupae and adults), and a

321 general trend for the difference between the male and female metabolome to

322 steadily increase throughout development. This increase in the extent of sexual

323 dimorphism largely reflects the broad pattern of phenotypic sexual dimorphism

324 increasing throughout development - phenotypically, males and females diverge

325 throughout development, ultimately resulting in dramatically different adult

326 phenotypes that are well-adapted to sex-specific roles (Darwin 1871).

328 This pattern of sexual dimorphism throughout development mirrors the results

329 of our transcriptomic analyses and is also consistent with other studies that

330 show an increasingly sexually dimorphic transcriptome throughout development

331 (Magnusson et al. 2011, Zhao et al. 2011, Ingleby et al. 2016). Our attempt to link 
332 previous transcriptomic data with the new metabolomic data was intended as

333 exploratory only. As such, further research could undoubtedly improve on this

334 straightforward comparison of two datatsets. More generally, the attempt to link

335 metabolomic and transcriptomic data highlights the availability of detailed -omic

336 data for D. melanogaster, and although to a lesser extent, other insect model

337 species as well. The increasing use of genomic, transciptomic and metabolomic

338 technology should mean that we can reach closer to a full understanding of how

339 genotype maps to phenotype - including the oft-overlooked steps between the

340 gene and the whole organism phenotype. This will be instrumental in future

341 sexual dimorphism research, since one of the key problems that a genotype-

342 phenotype map could address is how different phenotypes (i.e. sexes) are

343 produced from the same genes. With regards to the metabolome, this is unlikely

344 to be a simple linear map from gene to metabolite to phenotype. Recent research

345 has indicated, for instance, that environmental influences on the metabolome

346 could form a basis for interaction effects between genotype and phenotype

347 (Williams et al. 2015). Although complex, this suggests that more in-depth

348 metabolomic profiling - for example, within a quantitative genetic framework -

349 could be useful in understanding how different phenotypes are formed across

350 different contexts, including different sexes and developmental stages of

351 development. 
353

354

\section{5}

356

357

358

359

360

\section{Acknowledgements}

The authors are grateful to the Mass Spectrometry Facility at King's College London for their help processing the samples, and to Chris Mitchell for his help with using the ChemStation software. This work was supported by funding from the Swedish Research Council (2011-3701), the European Research Council (\#280632), and a Royal Society University Research Fellowship (all to E.H.M).

\section{References}

Baldwin JE, Krebs H. 1981. The evolution of metabolic cycles. Nature 291:381-32

Callier V, Hand SC, Campbell JB, Biddulph T, Harrison JF. 2016. Developmental changes in hypoxic exposure and responses to anoxia in Drosophila melanogaster. Journal of Experimental Biology 218:2927-2934

Chintapalli VR, Bratty MA, Korzekwa D, Watson DG, Dow JAT. 2013. Mapping an atlas of tissue-specific Drosophila melanogaster metabolomes by high resolution mass spectrometry. PLOS One 8:e78066

Colinet H, Larvor V, Laparie M, Renault D. 2012. Exploring the plastic response to cold acclimation through metabolomics. Functional Ecology 26:711-722

Darwin C. 1871. The descent of man and selection in relation to sex. John Murray, London, UK

Dean CAE, Teets NM, Kostal V, Simek P, Denlinger DL. 2016. Enhanced stress responses and metabolic adjustments linked to diapause and onset of migration in the large milkweed bug. Physiological Entomology 41:152-161 expression. Nature Reviews 8:689-698 
386 Hadfield JD. 2010. MCMC methods for multi-response generalised linear mixed models: the MCMCglmm R package. Journal of Statistical Software 33:1-22

Hahn DA, Denlinger DL. 2007. Meeting the energetic demands of insect diapause: nutrient storage and utilization. Journal of Insect Physiology 53:760-773 age, sex, and genotype on high-sensitivity metabolomics profiles in the fruit fly, Drosophila melanogaster. Aging Cell 13:596-604

Ingleby FC, Flis I, Morrow EH. 2015. Sex-biased gene expression and sexual conflict throughout development. Cold Spring Harbor Perspectives in Biology 7:a017632

Ingleby FC, Webster CL, Pennell TM, Flis I, Morrow EH. 2016. Sex-biased gene expression in Drosophila melanogaster is constrained by ontogeny and genetic architecture. Under review and available as a pre-print on bioRXiv, doi: http://dx.doi.org/10.1101/034728

Innocenti P, Morrow EH. 2010. The sexually antagonistic genes of Drosophila melanogaster. PLOS Biol 8:e1000335

Kochhar S, Jacobs DM, Ramadan Z, Berruex F, Fuerholz A, Fay LB. 2006. Probing gender-specific metabolism in humans by nuclear magnetic resonance-based metabonomics. Analytical Biochemistry 352:274-281

412 Kostal V, Korbelova J, Rozsypal J, Zahradnickova H, Cimlova J, Tomcala A, Simek

413 P. 2011. Long-term cold acclimation extends survival time at $0^{\circ} \mathrm{C}$ and modifies

414 the metabolomics profiles of the larvae of the fruit fly Drosophila melanogaster. PLOS One 9:e25025

417 Lande R. 1980. Sexual dimorphism, sexual selection, and adaptation to polygenic 418 characters. Evolution 34:292-305 
420 Laye MJ, Tran V, Jones DP, Kapahi P, Promislow DEL. 2015. The effects of age and

421 dietary restriction on the tissue-specific metabolome of Drosophila. Aging Cell

$422 \quad 14: 797-808$

423

424 Li Y, Zhang L, Chen H, Kostal V, Simek P, Moos M, Denlinger DL. 2015. Shifts in

425 metabolomics profiles of the parasitoid Nasonia vitripennis associated with

426 elevated cold tolerance induced by the parasitoid's diapause, host diapause and

427 host diet augmented with proline. Insect Biochemistry and Molecular Biology

$428 \quad 63: 34-46$

429

430 Magnusson K, Mendes AM, Windbichler N, Papathanos PA, Nolan T, Dottorini T,

431 Rizzi E, Christophides GK, Crisanti A. 2011. Transcription regulation of sex-

432 biased genes during ontogeny in the malaria vector Anopheles gambiae. PLOS

433 One 6:e21572

435 Michaud MR, Denlinger DL. 2007. Shifts in the carbohydrate, polyol, and amino

436 acid pools during rapid cold-hardening and diapause-associated cold-hardening

437 in flesh flies: a metabolomic comparison. Journal of Comparative Physiology B

$438 \quad 177: 753-763$

439

440 Overgaard J, Malmendal A, Sorensen JG, Bundy JG, Loeschcke V, Nielsen NC,

441 Homstrup M. 2007. Metabolomic profiling of rapid cold hardening and cold

442 shock in Drosophila melanogaster. Journal of Insect Physiology 53:1218-1232

443

444 Pawlowsky-Glahn V, Buccianti A. 2011. Compositional data analysis: theory and

445 applications. John Wiley and Sons, London, UK

446

447 Perry JC, Harrison PW, Mank JE. 2014. The ontogeny and evolution of sex-biased

448 gene expression in Drosophila melanogaster. Molecular Biology and Evolution

449 31:1206-1219 
451 Sarup P, Pedersen SMM, Nielsen NC, Malmendal A, Loeschcke V. 2012. The

452 metabolic profile of long-lived Drosophila melanogaster. PLOS One 7:e47461

454 Suhre K, Shin SY, Petersen AK, Mohney RP, Meredith D, Wagele B, Altmaier E, 455 CARDIoGRAM, Deloukas P, Erdmann J, Grundberg E, Hammond CJ, de Angelis

$456 \mathrm{MH}$, Kastenmuller G, Kottgen A, Kronenberg F, Mangino M, Meisinger C,

457 Meitinger T, Mewes HW, Milburn MV, Prehn C, Raffler J, Ried JS, Romisch-Margl

458 W, Samani NJ, Small KS, Wichmann HE, Zhai G, Illig T, Spector TD, Adamski J,

459 Soranzo N, Gieger C. 2011. Human metabolic individuality in biomedical and

460 pharmaceutical research. Nature 477:54-60

461

462 Williams S, Dew-Budd K, Davis K, Anderson J, Bishop R, Freeman K, Davis D, Bray

463 K, Perkins L, Hubickey J, Reed LK. 2015. Metabolomic and gene expression

464 profiles exhibit modular genetic and dietary structure linking metabolic

465 syndrome phenotypes in Drosophila. G3 5:2817-2829

467 Zhao M, Zha XF, Liu J, Zhang WJ, He NJ, Cheng DJ, Dai Y, Xiang ZH, Xia QY. 2011.

468 Global expression profile of silkworm genes from larval to pupal stages: toward a

469 comprehensive understanding of sexual differences. Insect Science 18:607-618 